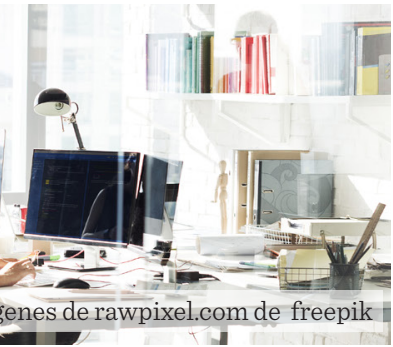

\title{
Integración, involucramiento y retroalimentación como antecedentes de la reflexividad en una dependencia gubernamental
}

\section{Integration, involvement and feedback as antecedents of reflexivity in a government agency}

\author{
Aurora Irma Máynez-Guaderrama', María Marisela Vargas-Salgado ${ }^{2 *}$
}

\section{RESUMEN}

Las organizaciones del sector público tienen como reto dar respuesta y solucionar las necesidades de la sociedad. Ante ese desafío, su gestión debe indagar la eficiencia de sus tareas. En esta vertiente, la reflexividad es una pieza clave, fundamental para el desempeño de los equipos de trabajo y útil para evaluar, buscar soluciones y tomar decisiones. El objetivo del presente estudio fue establecer, en una dependencia del gobierno mexicano, si la integración, el involucramiento y la retroalimentación, influyen en la reflexividad. El diseño fue cuantitativo, no experimental, transversal y causal. Se encuestó a 108 empleados. Los datos se recolectaron con un cuestionario auto administrado y como técnica de análisis se usó un modelo de ecuaciones estructurales con mínimos cuadrados parciales (PLS). Los resultados revelan que la integración, el involucramiento y la retroalimentación en esa dependencia tienen un efecto favorable y significativo en la reflexividad, reflejado en mayor medida en la búsqueda de acuerdos para alcanzar las metas laborales y la ejecución competente del trabajo; siendo la retroalimentación la variable de mayor influencia.

PALABRAS CLAVE: reflexividad, integración, involucramiento, retroalimentación, gobierno.

\section{ABSTRACT}

The challenge of public sector organizations is to respond and solve the needs of society. Faced with this hurdle, management must seek to improve task efficiency. In this regard, reflexivity is a key variable for team performance and useful for evaluating, seeking solutions, and making decisions. The aim of the present research is to determine if integration, involvement, and feedback variables have an influence on reflexivity, within the context of a government department. A quantitative, non-experimental, cross-sectional and causal design was employed. A sample of 108 employees was surveyed. Data were collected through a self-administered questionnaire and a model of structural equations with partial least squares (PLS) was used as an analysis technique. Results reveal that integration, involvement and feedback have a favorable and significant effect on reflexivity. In this context reflexivity is reflected to a greater extent in the search for agreements to achieve work goals and competent job execution. Overall, the variable exerting the greatest influence is feedback.

KEYWORDS: reflexivity, integration, involvement, feedback, government.

*Correspondencia: maria.vargas@uacj.mx/ Fecha de recepción: 6 de marzo de 2021/Fecha de aceptación:17 de septiembre de 2021/Fecha de publicación: 28 de enero de 2022.

${ }^{1}$ Universidad Autónoma de Ciudad Juárez, Instituto de Ingeniería y Tecnología, Departamento de Ingeniería Industrial y Manufactura, Ciudad Juárez, Chihuahua, México. ${ }^{2}$ Universidad Autónoma de Ciudad Juárez, Instituto de Ciencias Sociales y Administración, Departamento de Ciencias Administrativas, Av. Universidad y Av. Heroico Colegio Militar s/n, zona Chamizal, Ciudad Juárez, Chihuahua, México, C. P. 32300. 


\section{INTRODUCCIÓN}

La función de las dependencias de la administración pública es proporcionar servicios específicos a la sociedad (Coeto y Dávila, 2000), siendo uno de sus grandes retos dar respuesta y solucionar problemas en tiempo y forma (Bryson y col., 2014), y es en este contexto donde la reflexividad es un elemento necesario.

La reflexividad es un elemento fundamental que influye en todos los aspectos del quehacer de los grupos (Urbini y col., 2018), ya que permite que el conocimiento adquirido por las personas dentro y fuera de la empresa se aplique en ella de forma acertada en el día a día (Li y col., 2018). Además, ayuda en la búsqueda de alternativas para solucionar problemas, en la evaluación del desempeño y las estrategias y en la toma de decisiones (Gabelica y col., 2014). Desafortunadamente, las investigaciones sobre el tema son escasas (Urbini y col., 2018) y se demandan más estudios para comprender sus orígenes (De-Dreu, 2007).

Por su influencia en el aprendizaje, la reflexividad es relevante para el funcionamiento de los equipos (Schippers y col., 2012; 2014). Esta variable describe el grado en que piensan, reflexionan y adaptan estrategias, comportamientos, métodos de trabajo, objetivos $\mathrm{y}$ procesos cuando se enfrentan a circunstancias vigentes o anticipadas (Dreu, 2002; Schippers y col., 2012; 2014; Konradt y col., 2016; Yang y col., 2020). También se comprende como un proceso que cuestiona, monitorea y verifica la efectividad de hábitos, rutinas, metodologías, metas y procedimientos para promover el cambio y el aprendizaje (Farnese y col., 2016).

La reflexividad es una actividad crítica de procesamiento de información (Schippers y col., 2014) que va más allá de solo reflexionar en lo ocurrido (Urbini y col., 2018). En lo individual expresa la habilidad de demostrar en acciones el aprendizaje propio (Farnese y col., 2016), y en lo grupal fortalece las actividades de los equipos al mejorar el procesamiento de información (Schippers y col., 2014). En organizaciones poco flexibles, como las burocráticas, fomenta aceptación del cambio y actitudes innovadoras (Farnese y col., 2016).

La integración, el involucramiento y la retroalimentación influyen en la reflexividad de los grupos. La integración toma en cuenta la confianza y la cooperación para el logro de tareas compartidas (Lawrence y Lorsch, 1967; Younas y col., 2020). Del involucramiento de los empleados surge su colaboración y participación (Nadim y col., 2019) y es útil para reafirmar su compromiso tanto con los objetivos de la firma como con sus compañeros (de-Janasz y col., 2013). Y la retroalimentación permite un mejor desempeño (Moynihan y col., 2017; Hong, 2019), ya que ayuda a encauzar el comportamiento con las expectativas de la empresa (Battistelli y col., 2013).

Para lograr las metas organizacionales se requiere de la integración de los distintos equipos, y la confianza, el respeto y la armonía son ingredientes necesarios para conseguirla. La confianza tiene un importante papel en cualquier interacción, tanto a nivel personal como grupal (Kugler y col., 2007); ayuda a mejorar el intercambio e integración de conocimientos y la satisfacción con la actividad realizada (Bao y col., 2016; Brimhall, 2019). Por su parte, la armonía permite vivir experiencias positivas y de afecto, induce a percibir que cuando los compañeros expresan puntos de vista distintos lo hacen por motivos benéficos y lleva a balancear el comportamiento para mantener tanto la autonomía individual como la unidad de grupo (Ünal y col., 2017). En cuanto al respeto, es un atributo fundamental en las relaciones humanas; incluso se afirma que se valora más ser tratados con respeto que ser reconocidos o apreciados (Melhem y Al-Qudah, 2019).

La integración, a través de sus componentes implícitos, favorece la reflexividad en los equipos. La confianza les posibilita llevar a cabo actividades reflexivas (Widmer y col., 2009); aumenta la reflexividad al incidir tanto en la reflexión misma como en la acción y procesos subyacentes de la misma (De-Jong y Elfring, 2010). Además, disminuye el riesgo interperso- 
nal implícito, prerrequisito importante en las conductas reflexivas: las personas pueden hablar libremente y no sienten temor de hacerlo, pues no les preocupa que puedan aprovecharse de ellas (Widmer y col., 2009). Una cultura de respeto es necesaria cuando se busca lograr resultados que implican la actividad de distintas áreas, ya que ayuda a mantener relaciones satisfactorias entre las mismas (Melhem y AlQudah, 2019).

El involucramiento es una de las tareas más antiguas en la investigación del comportamiento organizacional (Glew y col., 1995). Este elemento es uno de los principales para el logro de los objetivos en las firmas (Nadim y col., 2019) y se considera esencial para fortalecer el compromiso y la satisfacción del personal (de-Janasz y col., 2013). En el gobierno, el involucramiento es necesario para asegurar la eficiente realización de las tareas (Tioumagneng y Njifen, 2019). Es un estado psicológico que consiste en la predisposición del sujeto para actuar en favor de algo (Tioumagneng y Njifen, 2019). A través de él se busca empoderar a los trabajadores para que participen en la toma de decisiones y se inserten activamente en las distintas áreas (Benson y col., 2013; Ramesh y Ravi, 2017). Al dar voz y voto a las personas se incrementa la posibilidad de que participen activamente en beneficio de la firma (Breeze y Wiepking, 2020).

El involucramiento incide en la reflexividad de los grupos, ya que implica un proceso de aprendizaje donde se cuestiona, revisa y evalúa la forma en que se desenvuelven y se piensa sobre los cambios necesarios para incrementar la efectividad (Reiter-Palmon y col., 2020). Estudios previos indican que en las organizaciones donde existe un alto grado de involucramiento se da una mayor comprensión cognitiva de la empresa y los empleados tienen la libertad para desenvolverse con autonomía y sin restricciones (Wallace y col., 2013).

Respecto a la retroalimentación, es un constituyente primario de la gestión del desempeño (Islami y col., 2018). Consiste en comunicar al personal los resultados asociados al cumplimiento de sus tareas, con la finalidad de que las mejoren y de ayudarlos al logro de objetivos (Doherty y Balzer, 1988; Hattie y Timperley, 2007). La información que reciben a través de la retroalimentación es de gran importancia y si se brinda oportunamente permite cambiar y tomar decisiones en el quehacer respectivo (Moynihan y col., 2017; Hong, 2019).

La retroalimentación también es necesaria para la reflexividad. Por una parte, genera confianza, incrementa la motivación y mejora el ejercicio laboral (Ling y col., 2016). Además, para lograr cambios en el aprendizaje y en la ocupación es necesario retroalimentar a los equipos; éstos deben ser receptivos a la misma, comprender su valor e involucrarse en actividades colaborativas donde la utilicen para mejorar la realización de sus tareas (Gabelica y col., 2014).

La reflexividad es un concepto clave para el logro de los objetivos de las organizaciones, representa un área de oportunidad, ya que fomenta el cambio y la innovación. En el último lustro se han efectuado investigaciones que abordan la reflexividad en diversos ámbitos. Por ejemplo, recientemente se indagó sobre su efecto en términos de cambio y gestión de recursos humanos (Ripamonti y col., 2020) como una condición que reduce las tres dimensiones del burnout (que es un estado de agotamiento físico, mental y emocional) (Chen y col., 2018), como un recurso útil para la innovación en grupos de cuidado de la salud (Reiter-Palmon y col., 2018) y como mediador entre las habilidades de gestión y la actuación en empresas de alta tecnología (Wu y col., 2017). En el entorno público, se detectaron análisis que valoran su efecto en la identidad de equipos en profesionales de cuidado social y de la salud comunitaria en Holanda (Hofhuis y col., 2018), como constructo moderador en los comportamientos de ayuda y desempeño en consultores de distintos proyectos (Fu y col., 2021). En ese periodo no se conocen investigaciones sobre la reflexividad en dependencias públicas mexicanas. 
El objetivo de este trabajo fue indagar si la integración, el involucramiento y la retroalimentación tienen influencia en la reflexividad en una dependencia pública del gobierno mexicano.

\section{MATERIALES Y MÉTODOS}

Se utilizó un diseño cuantitativo, no experimental, transversal y causal. Se encuestó a 108 sujetos de una dependencia pública del gobierno del estado de Chihuahua, México, cuya labor es garantizar justicia pronta y expedita; se reservan los datos para salvaguardar su confidencialidad. El trabajo de campo se desarrolló en las instalaciones de la dependencia en el mes de mayo de 2018, a solicitud del titular de la dependencia, asociado al requerimiento de un estudio de clima laboral como parte de un proceso de certificación.

Los cuestionarios se entregaron directamente a cada uno de los 108 participantes. Se explicó el propósito de la investigación y se indicó que la colaboración era voluntaria y anónima. La información solicitada incluía sexo, edad, escolaridad y antigüedad.

En la Figura 1 se presentan los constructos analizados. Para su operacionalización se revisaron en la literatura escalas disponibles. A partir de los hallazgos se diseñó el instrumen- to de medición, adaptando los ítems de cada variable de acuerdo con la escala propuesta por Patterson y col. (2005) (Tabla 1) y fueron valorados a través de una escala tipo Likert con 5 puntos de asignación de respuesta, que van desde "Totalmente en desacuerdo" hasta "Totalmente de acuerdo". En el instrumento se incluyeron 18 ítems: 5 de integración, 4 de involucramiento, 4 de retroalimentación y 5 de reflexividad. Ninguno de ellos se eliminó del modelo de medición.

De acuerdo con las recomendaciones de Anderson y Gerbing (1982), se siguió un procedimiento de dos etapas. En la primera se examinó el modelo de medición o externo; en la segunda se evaluó el modelo estructural o interno.

\section{Análisis del modelo de medición}

Validez convergente

Se inspeccionaron valores y significancia estadística de las cargas factoriales de cada uno de los ítems, sobre los que se recomienda 0.70 como punto de corte mínimo, ya que significa que existe mayor varianza compartida entre la variable latente y su medición (Fornell y Larcker, 1981); el coeficiente de alfa de Cronbach, mayor a 0.70, que evidencia la consistencia interna del constructo (Fornell y Bookstein, 1982); índice de fiabilidad compuesta (IFC), que a se-



- Figura 1. Variables estudiadas.

Figure 1. Studied variables. 
Tabla 1. Operacionalización de variables.

Table 1. Operationalization of variables.

\begin{tabular}{|c|c|}
\hline Constructo & Ítems \\
\hline \multirow{5}{*}{ Integración } & 1. Confianza entre los empleados de las distintas áreas. \\
\hline & 2. Colaboración efectiva entre las personas de las distintas áreas. \\
\hline & 3. Respeto entre las personas de las distintas áreas. \\
\hline & 4. Existe armonía. \\
\hline & 5. Disponibilidad para compartir información. \\
\hline \multirow{4}{*}{ Involucramiento } & 1. Los empleados son involucrados en la toma de decisiones que los afectan. \\
\hline & $\begin{array}{l}\text { 2. Se implementan cambios solo hasta que se toma en cuenta a las personas } \\
\text { involucradas en ello. }\end{array}$ \\
\hline & 3. Los empleados tienen voz y voto en las decisiones que afectan su trabajo. \\
\hline & 4. Se comparte ampliamente la información. \\
\hline \multirow{4}{*}{ Retroalimentación } & 1. Los empleados son retroalimentados respecto a la calidad de su trabajo. \\
\hline & 2. Los empleados saben qué tan bien están haciendo su trabajo. \\
\hline & 3. Es fácil para los empleados valorar la calidad de su desempeño. \\
\hline & 4. Se mide el desempeño de los empleados. \\
\hline \multirow{5}{*}{ Reflexividad } & 1. La forma de trabajar en equipo se cambia fácilmente para mejorar el desempeño. \\
\hline & $\begin{array}{l}\text { 2. Se buscan acuerdos respecto de los procedimientos para alcanzar las metas de } \\
\text { trabajo. }\end{array}$ \\
\hline & 3. Se reflexiona sobre el desempeño de los equipos de trabajo. \\
\hline & 4. Los objetivos se modifican de acuerdo con las circunstancias cambiantes. \\
\hline & 5. Se dedica tiempo para revisar los objetivos de trabajo. \\
\hline
\end{tabular}

Fuente: modificado a partir de Patterson y col. (2005).

mejanza del alfa de Cronbach, se aconseja un punto de corte mínimo de 0.70 como indicador de una mejor medida de consistencia interna, en virtud de que determina la magnitud en la cual los ítems se correlacionan y explican al constructo (Hair y col., 1999).

\section{Validez discriminante}

Análisis de varianza extraída (AVE). Con 0.50 como punto de corte, dado que asegura que al menos el $50 \%$ es explicada con los indicadores con los que se mide (Gefen y Straub, 2005). Estos parámetros se analizaron para cada uno de los constructos (Tabla 2). Se afirma que existe validez discriminante cuando las correlaciones entre los constructos del modelo son menores que las raíces cuadradas de los AVE de ellos (Fornell y Larcker, 1981).

Criterio heterotrait-monotrait. Se comparan las correlaciones heterotrait-heteromethod y las monotrait-heteromethod, y a partir del resultado se determina la validez discriminante; como referente, se utiliza un criterio de correlación que no exceda de 0.85 o 0.90, siendo el primero un criterio más conservador (Tabla 3) (Henseler y col., 2015).

\section{Análisis del modelo estructural}

Se revisaron los coeficientes paths, los valores $\mathrm{r}^{2} \mathrm{y} \mathrm{q}^{2}$. En la Figura 2 se presentan los primeros dos indicadores. Respecto de los paths estructurales, se recomienda que muestren valores mayores de 0.20 (Chin y Newsted, 1999). El coeficiente de determinación $\mathrm{r}^{2}$ permite identificar el grado en que el modelo explica los datos (Seidel y Back, 2009).

La calidad predictiva del modelo se establece con el indicador $q^{2}$ de Stone-Geisser. Los valores $q^{2}$, estimados por el procedimiento blindfolding, representan el grado en que el modelo path puede predecir los valores observados originalmente. Un valor de 0.35 se considera que, a través de 
Tabla 2. Validez discriminante de acuerdo con el criterio Fornell y Larcker.

Table 2. Discriminant validity according to the Fornell and Larcker criterion.

\begin{tabular}{|l|c|c|c|c|}
\hline \multicolumn{1}{|c|}{ Constructo } & Integración & Involucramiento & Retroalimentación & Reflexividad \\
\hline Integración & $0.840^{*}$ & & & \\
\hline Involucramiento & 0.559 & $0.847^{*}$ & & \\
\hline Retroalimentación & 0.524 & 0.457 & $0.885^{*}$ & \\
\hline Reflexividad & 0.614 & 0.535 & 0.602 & $0.897^{*}$ \\
\hline
\end{tabular}

Fuente: modificado a partir de datos de Smart PLS.

*Raíz cuadrada de AVE.

Tabla 3. Validez discriminante de acuerdo con Ratios Heterotrait-Monotrait.

Table 3. Discriminant validity according to the Heterotrait-Monotrait Ratios.

\begin{tabular}{|l|c|c|c|}
\hline \multicolumn{1}{|c|}{ Constructo } & Integración & Involucramiento & Retroalimentación \\
\hline Integración & & & \\
\hline Involucramiento & 0.610 & & \\
\hline Retroalimentación & 0.557 & 0.498 & \\
\hline Reflexividad & 0.642 & 0.567 & 0.651 \\
\hline
\end{tabular}

Fuente: modificado a partir de datos de Smart PLS.

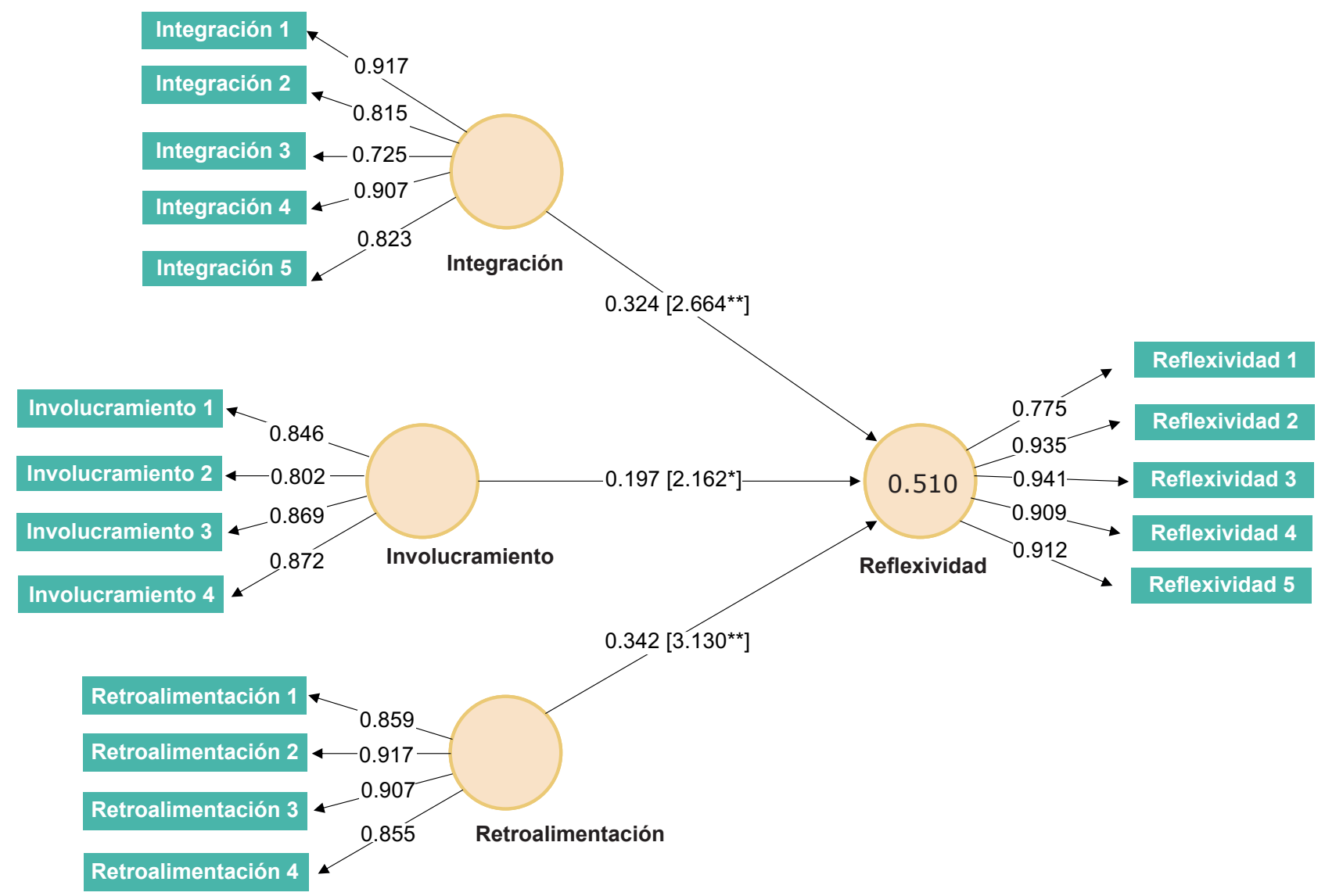

*Significativo con un alfa de Cronbach de 0.05, si $t>1.96$, para una prueba de dos colas.

**Significativo con un alfa de Cronbach de 0.01, si $t>2.576$, para una prueba de dos colas.

- Figura 2. Modelo contrastado.

Figure 2. Contrasted model. 
la redundancia, es una mejor media, y el punto de corte entre 0.02 a 0.35 muestra en qué medida el constructo exógeno tiene relevancia sobre la variable dependiente (Hair Jr y col., 2014).

La estadística descriptiva se analizó con ayuda del paquete estadístico para ciencias sociales (SPSS, por sus siglas en inglés: Statistical Package for the Social Sciences) (IBM, 2015) versión 23, y la inferencial con el software Smart PLS versión 3.0 (Ringle y col., 2015), que es una técnica de análisis que usa un modelo de ecuaciones estructurales con mínimos cuadrados parciales (PLS, por sus siglas en inglés: Partial Least Squares).

\section{RESULTADOS Y DISCUSIÓN}

De los 108 empleados participantes, 44 eran hombres (40.7 \%) y 64 mujeres (59.3 \%). Casi el $92 \%$ contaba con una escolaridad de carrera profesional concluida. El $43.5 \%$ tenía antigüedad en la dependencia de entre 1 y 3 años, y el $34.3 \%$ entre 4 y 7 años. El $84.3 \%$ reportó una edad entre 20 y 40 años (Tabla 4). En todos los casos los ítems superaron el punto de corte mínimo de las cargas factoriales (0.70), alcanzando valores entre 0.725 y 0.941 (Tabla 5), que fueron estadísticamente significativos a un nivel de confianza del $99.9 \%$ (valores $t$ entre 8.956 y 73.941, respectivamente).

En los cuatro constructos el valor alfa de Cronbach cumplió con la recomendación de ser mayor a 0.70: integración (0.896), involucramiento $(0.872)$, retroalimentación $(0.908)$ y reflexividad (0.938) . Asimismo, se revisó el IFC, que superó en todos los casos el punto de corte mínimo (0.70): integración (0.923), involucramiento (0.911), retroalimentación (0.935) y reflexividad (0.953), por lo que se demuestra que existe fiabilidad en el modelo de medición propuesto (Tabla 5).

En la diagonal de la Tabla 2 se presentan los valores de la raíz cuadrada de AVE y, bajo la misma, los valores de las correlaciones entre constructos. Como en ella se observa, en ninguno de los casos los valores de las correlaciones superan los de la raíz cuadrada de AVE (al revisar columna y fila), lo que indica que existe validez discriminante. El punto de corte fue superior a 0.50 en todos los casos: integración (0.706), involucramiento (0.718), reflexividad (0.804) y retroalimentación (0.783), que demuestra que existe validez convergente en el modelo de medición propuesto (Tabla 5).

Tabla 4. Características demográficas de los sujetos de estudio.

Table 4. Demographic characteristics of the study subjects.

\begin{tabular}{|c|c|c|c|}
\hline \multicolumn{2}{|r|}{ Características } & $n=108$ & $\%$ \\
\hline \multirow{2}{*}{ Sexo } & Hombres & 44 & 40.7 \\
\hline & Mujeres & 64 & 59.3 \\
\hline \multirow{5}{*}{ Edad } & Menos de 20 años & 1 & 0.9 \\
\hline & Entre 20 y 30 años & 53 & 49.1 \\
\hline & Entre 31 y 40 años & 38 & 35.2 \\
\hline & Entre 41 y 50 años & 13 & 12.0 \\
\hline & Más de 51 años & 3 & 2.8 \\
\hline \multirow{3}{*}{ Escolaridad } & Carrera profesional no terminada & 4 & 3.7 \\
\hline & Carrera profesional terminada & 99 & 91.7 \\
\hline & Otra & 5 & 4.6 \\
\hline \multirow{4}{*}{ Antigüedad } & Menos de 1 año & 17 & 15.7 \\
\hline & Entre 1 y 3 años & 47 & 43.5 \\
\hline & Entre 4 y 7 años & 37 & 34.3 \\
\hline & Más de 8 años & 7 & 6.5 \\
\hline
\end{tabular}


Tabla 5. Indicadores del modelo de medida.

Table 5. Measurement model indicators.

\begin{tabular}{|l|c|c|c|c|c|}
\hline \multicolumn{1}{|c|}{ Indicador } & Carga & Valor $\boldsymbol{t}$ & $\begin{array}{c}\text { Alfa de } \\
\text { Cronbach }\end{array}$ & $\begin{array}{c}\text { Índice de fiabilidad } \\
\text { compuesta }\end{array}$ & $\begin{array}{c}\text { Análisis de varianza } \\
\text { extraída (AVE) }\end{array}$ \\
\hline Integración 1 & 0.917 & $58.621^{*}$ & 0.896 & 0.923 & 0.706 \\
\hline Integración 2 & 0.815 & $15.056^{*}$ & & & \\
\hline Integración 3 & 0.725 & $8.956^{*}$ & & & \\
\hline Integración 4 & 0.907 & $46.356^{*}$ & & & \\
\hline Integración 5 & 0.823 & $19.472^{*}$ & & & 0.718 \\
\hline Involucramiento 1 & 0.846 & $15.485^{*}$ & 0.872 & & \\
\hline Involucramiento 2 & 0.802 & $11.108^{*}$ & & & \\
\hline Involucramiento 3 & 0.869 & $23.901^{*}$ & & & \\
\hline Involucramiento 4 & 0.872 & $38.618^{*}$ & & & \\
\hline Retroalimentación 1 & 0.859 & $27.276^{*}$ & 0.908 & & \\
\hline Retroalimentación 2 & 0.917 & $41.882^{*}$ & & & \\
\hline Retroalimentación 3 & 0.907 & $35.996^{*}$ & & & \\
\hline Retroalimentación 4 & 0.855 & $25.899^{*}$ & & & \\
\hline Reflexividad 1 & 0.775 & $10.625^{*}$ & 0.938 & & \\
\hline Reflexividad 2 & 0.935 & $52.499^{*}$ & & & \\
\hline Reflexividad 3 & 0.941 & $73.941^{*}$ & & & \\
\hline Reflexividad 4 & 0.909 & $36.029^{*}$ & & & \\
\hline Reflexividad 5 & 0.912 & $43.215^{*}$ & & & \\
\hline
\end{tabular}

*Significativo con un alfa de Cronbach de 0.001, si $t>3.291$, para una prueba de dos colas.

En la revisión de la validez discriminante se manejaron dos criterios: AVE y Heterotrait-Monotrait ratio. En ambos casos los resultados indican que existe validez (Tabla 2 y 3).

En cuanto al modelo estructural, dos de los paths estructurales cumplieron con la recomendación de tener valores superiores a 0.20 , integración $\rightarrow$ reflexividad (0.324) y retroalimentación $\rightarrow$ reflexividad (0.342); el tercero, involucramiento $\rightarrow$ reflexividad, mostró un valor marginal $(0.197)$ a ese punto de corte (Tabla 6). En todos los casos, los valores obtenidos fueron estadísticamente significativos.

El coeficiente de determinación $\mathrm{r}^{2}$ permitió identificar que el modelo explicó $51 \%$ (0.510) de la reflexividad. La calidad predictiva del modelo, establecida con el indicador $\mathrm{q}^{2}$ de StoneGeisser, indicó un valor de 0.08 para la integración, considerada como una relevancia predictiva entre baja y mediana; el involucramiento presentó baja relevancia predictiva $(0.03) \mathrm{y}$ la retroalimentación una relevancia predictiva mediana (0.11).

En la Tabla 6 se exponen las 3 hipótesis planteadas. Se aprecia que ninguna de ellas fue rechazada mediante el análisis estadístico. La integración ( $\left.\mathrm{H} 1, t=2.664^{* *}\right)$ y la retroalimentación (H3, $t=3.130 * *)$ impactaron positiva y significativamente en la reflexividad, a un nivel de confianza del $99 \%$, mientras que el involucramiento ( $\left.\mathrm{H} 2, t=2.162^{*}\right)$ influyó positiva y significativamente en la reflexividad, a un nivel de confianza del $95 \%$. Estos resultados indican que las tres relaciones son significativas, lo que abona favorablemente en términos teóricos y empíricos. Respecto de la teoría, se afirma que existen brechas que muestran que, no obstante su importancia, persiste una carencia de investigaciones sobre la reflexividad (Urbini y col., 2018) y sus orígenes (De-Dreu, 2007). Los datos encontrados en el presente estudio contribuyen en esa vertiente y proporcionan evidencia de la influencia de tres de sus antecedentes. En tér- 
Tabla 6. Prueba de hipótesis.

Table 6. Hypothesis testing.

\begin{tabular}{|l|c|c|c|}
\hline \multicolumn{1}{|c|}{ Hipótesis } & Coeficiente Path & Valor $\boldsymbol{t}$ & Resultado \\
\hline $\begin{array}{l}\text { H1. La integración influye positiva y significativamente en } \\
\text { la reflexividad. }\end{array}$ & 0.324 & $2.664^{* *}$ & No rechazo \\
\hline $\begin{array}{l}\text { H2. El involucramiento influye positivay } \\
\text { significativamente en la reflexividad. }\end{array}$ & 0.197 & $2.162^{*}$ & No rechazo \\
\hline $\begin{array}{l}\text { H3. La retroalimentación influye positivay } \\
\text { significativamente en la reflexividad. }\end{array}$ & 0.342 & $3.130^{* *}$ & No rechazo \\
\hline
\end{tabular}

*Significativo con un alpha de Cronbach de 0.05 , si $t>1.96$, para una prueba de dos colas.

**Significativo con un alfa de Cronbach de 0.01, si $t>2.576$ para una prueba de dos colas.

minos empíricos, se encontraron elementos que inciden significativamente sobre la reflexividad y que, por tanto, sirven para la gestión de entidades públicas en el contexto mexicano.

Entre las tres variables antecedentes, la retroalimentación es la que mayor influencia ejerce sobre la reflexividad (Figura 2). Los hallazgos coinciden con postulados previos que indican, que la retroalimentación puede modificar los pensamientos, comportamientos y resultados (Shute, 2008), y es útil cuando se desarrollan actividades de colaboración con propósitos de mejoramiento (Gabelica y col., 2014). En el contexto estudiado, la retroalimentación se refleja principalmente en el conocimiento de los empleados sobre qué tan bien realizan sus tareas (ítem retroalimentación 2, Tabla 5) y en la facilidad de valorar la calidad de las mismas (ítem retroalimentación 3 , Tabla 5 ). A la vez que, se confirma que la retroalimentación sobre ello les ayuda a mejorar sus actividades; si esto ocurre, aumenta la posibilidad de que piensen, reflexionen y adapten sus métodos de trabajo, objetivos y procesos conjuntamente con sus compañeros.

Acerca del vínculo entre la integración y la reflexividad, estudios previos indican que cuando los miembros comprenden plenamente la dirección y las prioridades de las metas, la confianza se incrementa y el desempeño mejora (Wagenvoort, 2020). En gran medida, ello se logra a través de la integración del grupo de trabajo. Estos hallazgos de la literatura científica confirman los planteamientos que muestran que el desarrollo de actividades reflexivas requiere de confianza y armonía (Widmer y col., 2009; De-Jong y Elfring, 2010; Ünal y col., 2017). Confianza, contribución, respeto, armonía y disponibilidad son piezas fundamentales para lograrlo. En la dependencia gubernamental examinada la integración favoreció la reflexividad (Figura 2) y se manifestó mayormente a través de la confianza entre personal de distintas áreas (ítem integración 1, Tabla 5) y en el reconocimiento de armonía entre ellos (ítem integración 4, Tabla 5). En síntesis, en los equipos integrados es mayor la posibilidad de que haya actividades reflexivas.

En cuanto a la relación entre el involucramiento y la reflexividad, los hallazgos empíricos coinciden con planteamientos que afirman que la reflexividad demanda procesos de aprendizaje donde se evalúa el desempeño y con base en los resultados se determinan los cambios requeridos para mejorar la efectividad grupal; para ello, se hace necesario el involucramiento y el empoderamiento y libertad para la toma decisional (Wallace y col., 2013; Breeze y Wiepking, 2020; Reiter-Palmon y col., 2020). En este estudio, el involucramiento se manifestó principalmente en la percepción de los empleados de que se comparte ampliamente la información (ítem involucramiento 4, Tabla 5) y se les otorga voz y voto en las decisiones que afectan su actividad (ítem involucramiento 3, Tabla 5). Luego, es posible afirmar que el involucramiento del equipo es útil en términos de reflexividad. 
Las restricciones de la presente investigación fueron su diseño transversal y que se llevó a cabo solamente en una dependencia del sector público mexicano. Esto restringe las posibilidades de generalización de resultados. Por ello, se llama a replicarlo en otras áreas gubernamentales y en otros países y, de ser posible, con alcances longitudinales y probabilísticos. Asimismo, se recomienda que se indague sobre las relaciones de la reflexividad con variables como la innovación, el liderazgo, el aprendizaje organizacional y la transferencia de conocimiento, particularmente en entidades del sector público.

La reflexividad es un factor relevante no solo para el logro de las metas de las empresas, sino también para las del gobierno. En este caso, se vinculan con la atención en tiempo y forma de las demandas ciudadanas de acuerdo con las atribuciones y responsabilidades de cada nivel (federal, estatal o municipal). Desafortunadamente, los estudios al respecto son escasos, particularmente en lo que se refiere a sus antecedentes.

\section{CONCLUSIONES}

La integración, el involucramiento y la retroalimentación de los trabajadores de la dependen- cia de gobierno analizada, son fundamentales para que existan actividades reflexivas. Esto significa que si reciben información sobre su desempeño, se implican en el logro de objetivos y laboran de forma integrada con sus compañeros, será mayor la posibilidad de que reflexionen en sus actividades como grupo, que establezcan acuerdos entre ellos y no solo se cuestionen su rendimiento, sino que busquen la forma de mejorarlo. En las dependencias del gobierno, la reflexividad puede llegar a convertirse en un aspecto nodal, porque las demandas ciudadanas son cambiantes y en ocasiones cada vez más complejas. La reflexividad lleva a los grupos de trabajo o equipos a pensar, reflexionar y adaptar sus objetivos, procesos y, en general, la forma en que desean cumplir sus objetivos; además, los hace cuestionarse si sus hábitos, rutinas y procedimientos son los adecuados. Para los investigadores de la materia, se abona en términos teóricos. Para los administradores públicos, estos hallazgos ayudan a valorar la importancia de las variables estudiadas en términos de reflexividad y dan la pauta para comenzar a impulsar este importante aspecto ante un entorno incierto y cada vez más demandante.

\section{REFERENCIAS}

Anderson, J. C. and Gerbing, D. W. (1982). Some methods for respecifying measurement models to obtain unidimensional construct measurement. Journal of Marketing Research. 19(4): 453-460.

Bao, G., Xu, B., and Zhang, Z. (2016). Employees' trust and their knowledge sharing and integration: the mediating roles of organizational identification and organization-based self-esteem. Knowledge Management Research \& Practice. 14(3): 362-375.

Battistelli, A., Montani, F., and Odoardi, C. (2013). The impact of feedback from job and task autonomy in the relationship between dispositional resistance to change and innovative work behaviour. European Journal of Work and Organizational Psychology. 22(1): 26-41.

Benson, G. S., Kimmel, M., and Lawler, E. E. (2013). Adoption of employee involvement practices: organizational change issues and insights. En A. B. Shani, W. A. Pasmore, R. W. Woodman, and D. A. Noumair (Eds.), Research in
Organizational Change and Development (pp. 233-257). Bingley, UK: Emerald Group Publishing Limited.

Breeze, B. and Wiepking, P. (2020). Different drivers: exploring employee involvement in corporate philanthropy. Journal of Business Ethics. 165(3): 453-467.

Brimhall, K. C. (2019). Inclusion is important ... but how do I include? Examining the effects of leader engagement on inclusion, innovation, job satisfaction, and perceived quality of care in a diverse nonprofit health care organization. Nonprofit and Voluntary Sector Quarterly. 48(4): 716-737.

Bryson, J. M., Crosby, B. C., and Bloomberg, L. (2014). Public Value Governance: Moving beyond traditional public administration and the new public management. $P u$ blic Administration Review. 74(4): 445-456.

Chen, J., Bamberger, P. A., Song, Y., and Vashdi, D. R. (2018). The effects of team reflexivity on psychological well-being in manufacturing teams. Journal of Applied Psychology. 103(4): 443-462. 
Chin, W. W. and Newsted, P. R. (1999). Structural equation modeling analysis with small samples using partial least squares. In R. H. Hoyle (Ed.), Statistical Strategies for Small Sample Research (pp. 307-341). Thousand Oaks, California: SAGE Publications.

Coeto, M. and Dávila, A. (2000). Efectividad en una dependencia de gobierno mexicana: evidencia para la modernización de la administración pública. Iztapalapa, Revista de Ciencias Sociales y Humanidades. 21(48): 119-134.

De-Dreu, C. K. W. (2007). Cooperative outcome interdependence, task reflexivity, and team effectiveness: A motivated information processing perspective. Journal of Applied Psychology. 92(3): 628-638.

De-Janasz, S., Forret, M., Haack, D., and Jonsen, K. (2013). Family status and work attitudes: An investigation in a professional services firm. British Journal of $M a-$ nagement.24(2): 191-210.

De-Jong, B. A. and Elfring, T. (2010). How does trust affect the performance of ongoing teams? The mediating role of reflexivity, monitoring, and effort. Academy of $\mathrm{Ma}$ nagement Journal. 53(3): 535-549.

Doherty, M. E. and Balzer, W. K. (1988). Cognitive feedback. In B. Brehmer and C. R. B. Joyce (Eds.), Advances in Psychology (pp. 163-197). North-Holland: Elsevier.

Dreu, C. K. D. (2002). Team innovation and team effectiveness: The importance of minority dissent and reflexivity. European Journal of Work and Organizational Psychology. 11(3): 285-298.

Farnese, M. L., Fida, R., and Livi, S. (2016). Reflexivity and flexibility: Complementary routes to innovation? Journal of Management \& Organization. 22(03): 404-419.

Fornell, C. and Bookstein, F. L. (1982). Two structural equation models: LISREL and PLS applied to consumer exitvoice theory. Journal of Marketing Research. 19(4): 440-452.

Fornell, C. and Larcker, D. F. (1981). Evaluating structural equation models with unobservable variables and measurement error. Journal of Marketing Research. 18(1): 39-50.

Fu, N., Flood, P. C., Rousseau, D. M., and Morris, T. (2021). Resolving the individual helping and objective job performance dilemma: The moderating effect of team reflexivity. Journal of Business Research. 129: 236-243.

Gabelica, C., van-den-Bossche, P., Segers, M., and Gijselaers, W. (2014). Dynamics of team reflexivity after feedback. Frontline Learning Research. 2(3): 64-91.

Gefen, D. and Straub, D. (2005). A practical guide to factorial validity using PLS-Graph: Tutorial and annotated example. Communications of the Association for Information
Systems. 16(1): 91-109.

Glew, D. J., O'Leary-Kelly, A. M., Griffin, R. W., and vanFleet, D. D. (1995). Participation in organizations: A preview of the issues and proposed framework for future analysis. Journal of Management. 21(3): 395-421.

Hair, J. F., Anderson, R. F., Tatham, R. L. y Black, W. C. (1999). Análisis multivariante (Quinta edición). Madrid: Prentice Hall Iberia. 828 Pp.

Hair Jr, J. F., Hult, G. T. M., Ringle, C., and Sarstedt, M. (2014). A primer on partial least squares structural equation modeling (PLS-SEM). United States of America: Sage publications. $307 \mathrm{Pp}$.

Hattie, J. and Timperley, H. (2007). The power of feedback. Review of Educational Research. 77(1): 81-112.

Henseler, J., Ringle, C. M., and Sarstedt, M. (2015). A new criterion for assessing discriminant validity in variance-based structural equation modeling. Journal of the Academy of Marketing Science. 43(1): 115-135.

Hofhuis, J., Mensen, M., Ten-Den, L. M., van-den-Berg, A. M., Koopman-Draijer, M., van-Tilburg, M. C., ..., and deVries, S. (2018). Does functional diversity increase effectiveness of community care teams? The moderating role of shared vision, interaction frequency, and team reflexivity. Journal of Applied Social Psychology. 48(10): 535-548.

Hong, S. (2019). A behavioral model of public organizations: Bounded rationality, performance feedback, and negativity bias. Journal of Public Administration Research and Theory. 29(1): 1-17.

IBM (2015). IBM SPSS Statistics for Windows (Versión 23.0) [Software de cómputo]. Armonk, NY: IBM Corp.

Islami, X., Mulolli, E., and Mustafa, N. (2018). Using management by objectives as a performance appraisal tool for employee satisfaction. Future Business Journal. 4(1): 94-108.

Konradt, U., Otte, K. P., Schippers, M. C., and Steenfatt, C. (2016). Reflexivity in teams: A review and new perspectives. The Journal of Psychology. 150(2): 153-174.

Kugler, T., Bornstein, G., Kocher, M. G., and Sutter, M. (2007). Trust between individuals and groups: Groups are less trusting than individuals but just as trustworthy. Journal of Economic Psychology. 28(6): 646-65\%.

Lawrence, P. and Lorsch, J. (1967). Differentiation and integration in complex organizations. Administrative Science Quarterly.12(1): 1-47.

Li, C. R., Li, C. X., Lin, C. J., and Liu, J. (2018). The influence of team reflexivity and shared meta-knowledge on the curvilinear relationship between team diversity and team ambidexterity, in Management Decision. [En línea]. Disponible en: https://doi.org/10.1108/MD-05-2017-0522. Fecha de consulta: 11 de noviembre de 2020. 
Ling, Y. L., Kanesan, A. G., and Ismail, F. (2016). Feedback environment and job motivation among the middle leaders of educational organizations. Journal of Education and Training. 3(1): 90-105.

Melhem, Y. S. and Al-Qudah, M. F. (2019). Work engagement: Trust and respect to engage your people. Indian Journal of Science and Technology. 12(17): 1-13.

Moynihan, D. P., Nielsen, P. A., and Kroll, A. (2017). Managerial use of performance data by bureaucrats and politicians. In O. James, S. R. Jilke, and G. G. van-Ryzin (Eds.), Experiments in Public Management Research: Challenges and Contributions (pp. 244-269). United Cambridge University Press.

Nadim, M., Fatima, S., Aroos, S., and Hafeez, M. H. (2019). Breach of psychological contract and job involvement: Does organizational cynicism mediates the relationship. Journal of Managerial Sciences. 13(3): 99-104.

Patterson, M. G., West, M. A., Shackleton, V. J., Dawson, J. F., Lawthom, R., Maitlis, S., ..., and Wallace, A. M. (2005). Validating the organizational climate measure: links to managerial practices, productivity and innovation. Journal of Organizational Behavior. 26(4): 379-408.

Ramesh, N. and Ravi, A. (2017). Determinants of total employee involvement: a case study of a cutting tool company. International Journal of Business Excellence. 11(2): 221-240.

Reiter-Palmon, R., Kennel, V., Allen, J., and Jones, K. J. (2018). Good Catch! Using Interdisciplinary Teams and Team Reflexivity to Improve Patient Safety. Group \& Organization Management. 43(3): 414-439.

Reiter-Palmon, R., Leone, S., Murugavel, V., and Allen, J. A. (2020). Fostering Effective debriefs: The integral role of team reflexivity. In L. M. Annika, A. A. Joseph, and L. W. Nale (Eds.), Managing Meetings in Organizations (pp. 93-109). Bingley, UK: Emerald Publishing Limited.

Ringle, C. M., Wende, S., and Becker, J. M. (2015). Smart PLS 3 (Versión 3.0) [Software de cómputo]. Bönningstedt, Pinneberg: SmartPLS.

Ripamonti, S. C., Galuppo, L., Provasoli, G., and Benozzo, A. (2020). Unmasking Reflexivity in HR Managers During the COVID-19 Lockdown in Italy. Frontiers in Psychology. 11(3270).

Schippers, M. C., Edmondson, A. C., and West, M. A. (2014). Team reflexivity as an antidote to team informationprocessing failures. Small Group Research. 45(6): 731-769.

Schippers, M. C., West, M. A., and Dawson, J. F. (2012). Team reflexivity and innovation: the moderating role of team context. Journal of Management. 41(3): 769-788.

Seidel, G. and Back, A. (2009). Success factor validation for global ERP programmes. Paper presented at the 17th European Conference on Information Systems, Verona. [En línea]. Disponible en: https://www.alexandria.unisg. ch/213715/1/ecis2009-0098.pdf. Fecha de consulta: 3 de diciembre de 2020.

Shute, V. J. (2008). Focus on formative feedback. Review of Educational Research. 78(1): 153-189.

Tioumagneng, A. and Njifen, I. (2019). Employee involvement in the public administrative sector: an exploration of the engagement drivers in Cameroon. International Review of Administrative Sciences. 86(4): 765-781.

Ünal, A. F., Chen, C. C., and Xin, K. R. (2017). Justice climates and management team effectiveness: The central role of group harmony. Management and Organization Review. 13(4): 821-849.

Urbini, F., Callea, A., Chirumbolo, A., Talamo, A., Ingusci, E., and Ciavolino, E. (2018). Team performance in the Italian NHS: the role of reflexivity. Journal of Health Organization and Management. 32(2): 190-205.

Wagenvoort, M. (2020). A plea for team reflexivity in organizations a three-way interaction model with team reflexivity, goal clarity and participative leadership on team performance, en Repositorio Institucional- Universidad de Utrecht. [En línea]. Disponible en: https://dspace.library. uu.nl/handle/1874/397904. Fecha de consulta: 25 de noviembre de 2020.

Wallace, J. C., Butts, M. M., Johnson, P. D., Stevens, F. G., and Smith, M. B. (2013). A multilevel model of employee innovation: Understanding the effects of regulatory focus, thriving, and employee involvement climate. Journal of Management. 42(4): 982-1004.

Widmer, P. S., Schippers, M. C., and West, M. A. (2009). Recent developments in reflexivity research: A review. Psychology of Everyday Activity. 2(2): 2-11.

Wu, W. Y., Amaya Rivas, A., and Chen, Y. C. (2017). The role of team reflexivity as a mediator between project management skills, task familiarity, procedural justice, and product performance. Journal of Management \& Organization. 25(6): 876-895.

Yang, M., Schloemer, H., Zhu, Z., Lin, Y., Chen, W., and Dong, N. (2020). Why and when team reflexivity contributes to team performance: A moderated mediation model. Frontiers in Psychology. 10(3044).

Younas, A., Wang, D., Javed, B., and Zaffar, M. A. (2020). Moving beyond the mechanistic structures: The role of inclusive leadership in developing change-oriented organizational citizenship behaviour. Canadian Journal of Administrative Sciences/Revue Canadienne des Sciences de l’Administration. 1(20): 1-11. 